Cahiers d'études africaines

\title{
Le non-standard entre norme endogène et fantasme d'unicité
}

L'épopée abidjanaise et sa polémique intrinsèque

\section{Katja Ploog}

\section{(2) OpenEdition}

\section{Journals}

Édition électronique

URL : http://journals.openedition.org/etudesafricaines/103

DOI : 10.4000/etudesafricaines. 103

ISSN : $1777-5353$

\section{Éditeur}

Éditions de l'EHESS

Édition imprimée

Date de publication : 1 janvier 2001

Pagination : 423-442

ISBN : 978-2-7132-1394-6

ISSN : 0008-0055

\section{Référence électronique}

Katja Ploog, «Le non-standard entre norme endogène et fantasme d'unicité », Cahiers d'études africaines [En ligne], 163-164 | 2001, mis en ligne le 31 mai 2005, consulté le 05 février 2021. URL: http://journals.openedition.org/etudesafricaines/103; DOI : https://doi.org/10.4000/etudesafricaines. 103 


\section{Katja Ploog}

\section{Le non-standard entre norme endogène et fantasme d'unicité}

\section{L'épopée abidjanaise et sa polémique intrinsèque}

\section{Vivre le français en Afrique : problématique}

Glottophagie francophone et appropriation du français

Dans l'Afrique postcoloniale, l'option souvent considérée comme la plus «pragmatique » en matière de politique linguistique a consisté à maintenir l'ancienne langue coloniale comme langue officielle (seule ou conjointement à une ou plusieurs langues «nationales »). Parmi les pays ayant adopté cette solution, on recense treize ex-colonies françaises, y compris la Côted'Ivoire. À l'opposé, les tentatives de promouvoir les langues africaines ont souvent été accueillies avec méfiance par les Africains: la peur d'un enseignement au rabais et celle de l'isolement dans la communauté internationale laissaient apparaître le français comme le choix le plus rentable, du point de vue institutionnel autant qu'individuel. Or, la diffusion du français pose apparemment problème : Chaudenson (1989: 357) retire de son diagnostic des situations francophones que le français, dans la plupart des pays, souffre d'un déficit aigu de corpus face à un status prépondérant.

Cependant, en trente années d'indépendance, les locuteurs africains se sont littéralement appropriés l'ancienne langue coloniale, en la façonnant selon leurs besoins. Dès lors, le français n'est plus véritablement superposé aux langues nationales, mais vient se ranger parmi elles, au sein de la configuration plurilingue du pays. Aujourd'hui, d'après les linguistes, l'émergence d'une norme endogène est avérée. Un bon résumé de la représentation qu'en ont les observateurs est fourni par cette description de Tabi-Manga (1992: 115) :

«L'analyse de la variation du français en Afrique fait apparaître une dichotomie : français littéraire/français véhiculaire. D’une façon générale, le français littéraire reste encore nettement dominant dans les usages quelle que soit la situation de communication. Ce français littéraire s'oppose à celui véhiculaire et vivant pratiqué par une large majorité de locuteurs au marché, au stade, dans les bureaux et autobus. 
Cette langue vivante et dynamique, colorée et adaptée à toutes les situations de communication est partout pourchassée et farouchement combattue parce qu'elle est porteuse d'incorrections et d'impuretés qui menaceraient la norme. »

De toute évidence, l'auteur de ces propos raisonne plus en tant que locuteur d'une variété acrolectale qu'en tant que linguiste lorsqu'il juge le français «littéraire » dominant, tout en constatant, une ligne plus loin, que le français «véhiculaire » est omniprésent (mais pas reconnu) : en le niant, il le combat, lui aussi. Tabi-Manga semble faire la double confusion entre registre comme emploi spécifique à un type de situation et variété sociale — «vivante» et « colorée »! - en plus de celle entre français littéraire et français standard: pourquoi l'appellation véhiculaire pour l' «autre » variété, si les deux conviennent dans toutes les situations de communication? Comment croire que le français littéraire reste dominant dans les usages quelle que soit la situation, quand on connaît les conditions locales d'acquisition et d'emploi prédominantes ?

Si « la norme africaine s'impose d'elle-même, naturellement, grâce à l'usage du plus grand nombre » (Dumont 1991: 16), rien ne paraît moins clair que son étendue (territoriale) et ses limites (structurales). Quelle est donc cette norme africaine ? À une échelle d'observation plus modeste qui est l'exemple de la situation abidjanaise - dans son contexte, qui est le plurilinguisme ivoirien — je m'interrogerai sur la difficulté d'établir une norme locale distincte de la norme de référence standard, et sur le malaise d'avoir à nommer et à classer l'hétérogénéité qu'on observe.

\section{Le français en Côte-d'Ivoire}

Le français coexiste en Côte-d'Ivoire avec plus de 60 langues ethniques de plus ou moins grande diffusion dont aucune n'est réellement dominante. Mais sa situation diffère de celle observée dans d'autres pays de tradition coloniale similaire. Selon Simard (1994), le sentiment d'appartenance nationale serait aujourd'hui plus fort chez les Ivoiriens que celui de l'appartenance ethnique. Fiers de la réussite de leur patrie et heureux d'avoir évité (jusqu'à ce jour) des affrontements comme au Rwanda ou au Liberia, les jeunes intellectuels reprennent avec ferveur les arguments de Senghor (1988), en soulignant plus particulièrement l'aspect fédérateur du français.

Au cours de mes enquêtes, j'ai pu constater que certains groupes ethniques sont en effet prêts à sacrifier leur langue au profit du véhiculaire exogène, mais non au profit d'une autre langue nationale ${ }^{1}$. En corollaire, la complémentarité fonctionnelle des langues est restée un postulat pour se dissoudre dans une bilingualité soustractive où les langues ethniques laissent progressivement leur place au français.

1. Langue nationale ici dans le sens originaire du pays. 
Les principales classifications des différentes variétés ${ }^{2}$ de français en Côte-d'Ivoire ont été élaborées par Duponchel $(1974,1979)$ et par Lafage (1978b, 1984). De nombreux auteurs se sont basés, plus ou moins explicitement, sur ces classifications ${ }^{3}$, fondées sur des critères généralement sociologiques ou fonctionnels. La description structurale des variétés distinguées n'est cependant que très peu avancée. Bien que la nomination ${ }^{4}$ paraisse tout sauf homogène, les observateurs (linguistes) conviennent d'une articulation des pratiques linguistiques en continuum, allant du français standard aux langues ivoiriennes.

La variété locale la plus «originale » porte dans la littérature le nom de français populaire d'Abidjan ou de Côte-d'Ivoire (FPA ou FPI respectivement). Pour Crespo-Meunier (1991), le FPA est un démodialecte appartenant à l'aire d'intercompréhension du français. Hattiger (1983) avait conclu auparavant que le fonctionnement du groupe nominal en FPA ressemblait à celui des Pidgins. Dès le début des années soixante-dix, Duponchel (1971) laissait entrevoir une évolution vers un créole local. Or, cette éventualité n'a visiblement incité personne à tenter d'y voir plus clair.

Il y a trente ans encore, les regards étaient souvent dirigés vers la Côted'Ivoire, émergente et prospère à l'époque, avec une place particulière accordée à sa capitale Abidjan, métropole phare de l'Afrique de l'Ouest; cet intérêt a considérablement décru depuis, autant en linguistique qu'en sciences sociales. Parfois, on ne peut s'empêcher de penser qu'il est plus commode de se « cacher » derrière des analyses anciennes parce que la situation actuelle paraît bien plus complexe encore, plus délicate à appréhender.

\section{Côté terrain : vivre Abidjan}

\section{Comment plébisciter une langue}

En 1933, lorsque l'administration française choisit le site au bord de la lagune comme nouvelle capitale de la Côte-d'Ivoire, Abidjan n'est qu'un village ebrie ${ }^{5}$; dans les années quatre-vingt-dix, près d'un tiers de la population du pays s'y trouve concentrée ${ }^{6}$. Le taux de croissance le plus important a été observé dans les années cinquante et soixante, lorsque la natalité était la plus forte et les migrations les plus massives ; les mouvements concernaient en grande partie des populations défavorisées, pas ou peu scolarisées,

2. Faute de mieux, j'utilise le terme variété comme générique, tel lecte, employé par Cécile Canut. Pour rendre justice aux descriptions d'autres auteurs, j'utiliserai cependant leur terminologie.

3. Comme Kokora (1983), Partmann (1981), Derive (1986), Manessy (1992).

4. Pour une analyse détaillée, voir ma thèse (Ploog 1999a).

5. Ethnie du groupe kwa.

6. En 1990, pour la première fois, la majorité de la population ivoirienne est urbaine, face à $1,6 \%$ en 1920 . 
des origines ethnique et nationale les plus diverses, cherchant à la ville de meilleures conditions économiques.

La violence des vagues migratoires et l'urbanisation sauvage qui s'ensuivait ont rapidement engendré une promiscuité marquée, favorable à l'échange interethnique jusque dans la sphère privée. Plus des trois quarts des Abidjanais habitent dans des cours, regroupement de logements similaire à la concession traditionnelle de la famille élargie, mais sur un espace plus confiné et les liens familiaux en moins : les logements sont disposés autour de la cour commune où se trouvent sanitaires, fourneaux et, dans les cours plus riches, des espaces réservés à l'artisanat, à la lessive, ou bien aux jeux. Le logement courant (Dubresson \& Yapi Diahou 1988: 1095) comporte une seule pièce, sinon deux. L'éventail de catégories socioprofessionnelles représenté dans ce type d'habitat est très large: bien que les cadres et manœuvres cohabitent rarement, on y trouve des propriétaires enseignants, habitant leur cour même, qui louent des logements à des ouvriers. Les relations familiales et ethniques ne peuvent que se diluer dans une population néo-urbaine à la recherche de ses marques.

Cette «macrocéphalie » (Marguerat 1979: 71) qui affecte désormais la Côte-d'Ivoire constitue l'un des vecteurs de la dégradation des conditions de vie - et non pas, comme espéré, un moteur au développement moderne. Face au désengagement de l'État, la précarité est quasi générale. En effet, c'est une économie parallèle, le fameux «secteur informel», qui permet aux trois quarts de la population de (sur)vivre.

On devine à quelle vitesse les contacts linguistiques se multiplient dans ce cadre de vie - pour imposer en corollaire l'habilitation urgente d'un véhiculaire. La communauté agit en tant que locuteur en puissance quand elle choisit sa langue : la langue favorisée, autant chez le locuteur que dans la communauté, est celle qui permet la prise en charge du plus grand nombre de relations - et celle qui permet la réussite en ville (Manessy 1990 : 17). Jusqu'alors, seuls les Malinké (groupe mande nord) ressortaient de la diversité ethnique ivoirienne en raison de leur plus grande mobilité (due à leur activité commerciale), qui a favorisé le développement de leur langue, le diula, comme moyen de communication interethnique à l'échelle du pays. Mais le diula restait trop étroitement associé aux populations du Nord, immigrées et surtout musulmanes : les populations de la bande côtière (akan/kwa et kru) sont de confession chrétienne et ont bénéficié — au moins partiellement - d'une scolarisation en français. En outre, le rêve de faire fortune en ville risquait de se trouver vite étouffé avec comme seul bagage le diula, qui ne permettait pas de promotion sociale au-delà du secteur «informel ». Celui qui s'inscrivait pleinement dans le monde moderne ne pouvait alors plus choisir que le français : «Au fond, pourquoi hésiter à utiliser le langage moderne de notre monde contemporain ? N'avons-nous pas accepté sa voiture, ses chaussures, en un mot tous ses outils et conforts... » (Aké Loba, cité par Duponchel 1979: 396). 


\section{Construire une identité commune}

Si le degré d'intégration d'un migrant est proportionnel à son maniement de la langue de la ville, la constellation initiale de plurilinguisme évolue parallèlement à la constitution de la communauté urbaine. Mais si le français a progressivement supplanté les langues autochtones, cette dynamique a été accompagnée par sa propre diversification. Lafage (1978a : 61) décrit l'importance du français populaire d'Abidjan (FPA) au milieu des années soixante-dix dans les termes suivants : «La vie quotidienne met, en milieu urbain, tout francophone local en contact avec ce parler $»^{7}$; aujourd'hui on pourrait dire que la vie urbaine met, à Abidjan, tout habitant en contact avec lui — et parfois même exclusivement.

Depuis 1990, le taux de scolarisation stagne, il aurait même tendance à régresser ; la déscolarisation, sur laquelle aucune statistique n'est publiée, reste le lot commun de la majorité des écoliers ${ }^{8}$ abidjanais; un coût de scolarisation trop élevé pour une grande partie des familles et des capacités d'accueil largement insuffisantes en sont responsables. Par conséquent, au bout de quelques années de scolarisation, beaucoup de jeunes enfants se trouvent dans la rue, à exercer une activité rémunérée pour apporter une contribution financière au fonctionnement du «ménage », notion très relative dans ces quartiers où le regroupement de la famille élargie sous un même toit reste réservé aux franges privilégiées de la population. Au dehors, le groupe de pairs constitue un facteur d'intégration fondamental, à travers notamment la revendication d'une sous-variété du véhiculaire (fortement marqué par le mélange, et l'hybridation ${ }^{9}$ ) d'une part, et d'autre part la constitution de réseaux sociaux. Les Abidjanais natifs composent aujourd'hui la majorité de la population; la très grande majorité parmi eux a grandi avec la langue urbaine, dont les contours restent à définir.

Inutile d'insister sur le fait que les conditions de vie que connaissent les Abidjanais, autant qu'elles sont très extrêmes, ne sont pas les mêmes dans le reste du pays. La véhicularité du français tout comme sa vernacularisation progressive restent, du moins dans un premier temps, restreintes à la métropole. Mais la dynamique est engagée et l'emprise du français non standard ne se limite pas à la seule ville d'Abidjan, car malgré tout, beaucoup d'Abidjanais continuent à maintenir des relations avec leur région d'origine, la mobilité croissante aidant. Mais cette expansion horizontale, souvent postulée au cours des vingt dernières années, n'a toujours pas été établie par les enquêtes appropriées.

7. C'est moi qui souligne.

8. Selon ChaUdENSON (1993 : 402), le taux d'abandon pendant les quatre premières années de scolarisation s'élèverait, en 1993, à 30-50\% d'une classe d'âge, le taux d'enfants sachant correctement lire et écrire au bout de la même période ne dépasserait pas les $5 \%$.

9. D'après le terme consacré par Lafage, désignant un métissage impliquant plus de deux langues dans les procédés néologiques. 


\title{
Côté observation : de la variabilité à la norme endogène
}

\author{
La dynamique ivoirienne vue de l'extérieur
}

La subversion de la langue importée fut la réponse ivoirienne à la mainmise postcoloniale ; le développement d'un continuum brouille les pistes de la description linguistique.

Le tout premier pas en direction de la reconnaissance des particularités locales est accompli par leur droit de cité. Les premières recherches menées, depuis la fin des années soixante, s'inscrivaient dans la perspective d'améliorer l'enseignement du français. Duponchel (1971 : 14) note le caractère très marginal encore du monolinguisme français en Côte-d'Ivoire :

«[...] précisons que nous excluons de notre propos les écoliers ivoiriens issus de milieux familiaux uniquement francophones et ne parlant aucune langue africaine. Dans certaines écoles d'Abidjan, ces élèves constituent la majorité de l'effectif: enfants de fonctionnaires, de familles urbanisées depuis plus d'une génération, de ménages franco-ivoiriens. [...] Bien que leur nombre soit en rapide augmentation, ils ne constituent encore, à l'échelle du pays, qu'une infime minorité. »

Le français restait encore un « outil de communication dont ne disposent qu'une minorité d'Ivoiriens, ceux qui ont en général le plus haut statut social » (Duponchel 1974: 140). Mais si «leur nombre [était] en rapide augmentation » alors qu'une dissolution des hiérarchies sociales n'était pas en vue, la variété en question ne pouvait être le français standard. Dès 1971, Duponchel (1971 : 16) signalait une possible autonomisation pour le FPA :

« Il paraît certain qu'à brève échéance de jeunes Ivoiriens nés de parents d'ethnies différentes auront comme langue maternelle cette variété de français. La nécessité d'exprimer, grâce à cet outil, toute la diversité et la complexité de l'expérience vécue amènera des enrichissements [de ce parler]. Ainsi se constituerait un véritable créole à base de français, parallèle au français officiel. »

Il envisage l'éventualité que le développement « [débouche] sur une rupture de l'intercompréhension entre francophones» (id. 1974: 142). L'authentique se mue alors en superflu : «Ce français perdrait alors sa qualité de langue internationale sans pour autant présenter d'avantages notables sur les langues africaines maternelles, hormis peut-être une plus grande ouverture de son lexique aux concepts des sociétés industrialisées. »

Le FPA ne doit pas avoir d'avenir. À l'aube des années quatre-vingt, les observateurs du terrain ivoirien deviennent alors plus optimistes : ils ne prédisent guère d'avenir aux variétés locales de français. Lafage (1978b) estime qu'il serait plus approprié de parler de niveaux d'acquisition que de variétés, car les approximations diverses des locuteurs évoluent d'un «"pseudo-sabir franco-africain" [...] jusqu'à une forme stabilisée ». Duponchel (1979: 410) explique : 
«Compte tenu de l'emprise des mass media et des efforts de scolarisation, il nous paraît exclu, dans les circonstances actuelles, que puisse s'établir un véritable pidgin à base de français. En contact permanent avec des variétés de français à statut plus élevé, les formes rudimentaires du français tendent, chez un même locuteur, à se rapprocher des formes valorisées. »

On assisterait ainsi à un nivellement progressif par le haut — qui se fait néanmoins attendre - : si plus d'un tiers des Ivoiriens parlent le français dès 1980 (Lafage 1978b), il ne s'agit pas pour la plupart du français standard, mais du FPI.

Par ailleurs, la diversification du français génère en corollaire une conscience linguistique grandissante, et le droit de cité du non-standard va en croissant à partir du milieu des années soixante-dix, où les premières manifestations publiques en non-standard sont recensées, ainsi la chronique « français de Moussa » publiée dans l'hebdomadaire Ivoir'Dimanche :

«La difficulté qu'on éprouve à définir la norme endogène provient de ce que la normalité qui la fonde n'est perçue que dans l'interaction même. Elle ne donne qu'exceptionnellement lieu à des représentations conscientes, lorsqu'elle acquiert une fonction emblématique ou identitaire. Elle tend alors à se stéréotyper, à devenir prescriptive, donc descriptible : c'est ainsi que s'est constitué en Côte-d'Ivoire le "français de Moussa", version littéraire de l'usage populaire ivoirien » (Manessy 1994: 218).

L'appellation choisie par Manessy est aussi rare que juste. Le plus souvent, le français de Moussa est décrit comme forme caricaturale du français populaire :

«Il s'agit d'énoncés pensés en français et réécrits par des intellectuels qui en exagèrent à plaisir les déformations pittoresques, pour la délectation de lecteurs euxmêmes instruits. Le but ouvertement visé est le comique, mais ce comique n'existe en fait que pour ceux qui possèdent au moins le français central comme point de comparaison »(Duponchel 1974: 146).

L'effet humoristique (voulu) de ces chroniques prête à confusion : l'intention des auteurs est nullement la caricature, et ce n'est pas l'interprétation des lecteurs non plus. Le décalage entre le français de Moussa et le FPA semble résulter du fait de la transcription d'un côté et de l'origine sociale des auteurs de l'autre. De fait, la chronique de Moussa, inoffensive et artificielle pour les linguistes, menaçant pour le modèle linguistique des écoliers selon les enseignants, constitue avant tout la première revendication publique d'une spécificité linguistique ivoirienne, de la part des intellectuels.

Un autre exemple en constitue l'excellent film Bronx Barbès ${ }^{10}$, sur les écrans en fin d'année 2000: si l'empreinte du contexte social abidjanais (le décor) me paraît extrêmement précise, les échanges verbaux n’y sont

10. Un film d'Éliane de Latour. 
pas plus authentiques que dans n'importe quelle autre production artistique, et ne peuvent à mes yeux rendre compte de l'abidjanais qu'à ce titre, à savoir en retenant, comme par un filtre, des clichés de l'argot local.

\section{L'articulation du continuum : des déviances aux représentations de la Norme}

L'habilitation d'un usage local n'est pas interprétée d'entrée comme un découragement face à la norme centrale; l'accent est mis sur le fait que le français «n'est plus tout à fait senti comme un corps étranger [...]. Alors qu'autrefois un souci exagéré de purisme se manifestait chez les élèves et étudiants [...], il n'est pas rare actuellement d'entendre revendiquer dans ces mêmes milieux le droit de parler le français à l'africaine » (Duponchel 1974 : 141).

Comme le souligne Duponchel (1979: 405), «c'est la face cachée de l'iceberg qui est numériquement et socialement la plus importante ${ }^{11}$, iceberg composé des locuteurs ayant une pratique du français qui repose sur une connaissance exclusivement orale. Or, la plupart des études ont été menées à partir de corpus écrits, de copies d'élèves notamment. Les seuls travaux effectués hors du milieu institutionnel (Hattiger 1983; CrespoMeunier 1991) ont pour base des échanges contraignants, exclusivement asymétriques, peu propices, malgré de réelles précautions observées, aux productions non surveillées: le point commun de tous les corpus est l'omniprésence de la norme. Crespo-Meunier (1991) définit le FPA comme démodialecte, parler acquis par imprégnation, en l'opposant aux langages complexifiés, qui nécessiteraient un second apprentissage, raisonné. Il n'est que conséquent de sa part de conclure par ailleurs qu'il s'agit d'un "parler sans grande cohésion syntaxique » (id. 1998: 307).

Dans ce contexte, l'africanisme redevient écart de la norme, « manifestation, certes défaillante mais irréfutable, de la mise en place d'un système approximatif de communication» (Dumont 1991: 120), et représente une étape intermédiaire dans l'acquisition du français standard:

«Il est bien évident que toute politique fondée sur une éventuelle codification du français d'Afrique suppose une reconnaissance implicite, de facto, d'un décalage entre les situations hexagonales et africaines. Or, l'Afrique n'est peut-être pas encore assez mûre linguistiquement pour couper le cordon ombilical [...]» (ibid. : 15).

Vue partielle ou partiale ? La revendication d'une norme endogène surprend d'autant plus : «En Afrique, dans l'usage mésolectal qu'il faut maintenant prendre pour référence, c'est l'efficacité de la communication qui prime et non la conformité à la norme » (id. 1993 : 477). Tout comme pour

11. «Partout où il joue le rôle d'un véhiculaire, partout où il se vernacularise, le français qui se généralise est celui de la rue »(Dumont 1991 : 109). 
n'importe quel locuteur de n'importe quelle langue en situation informelle, serait-on tenté d'ajouter.

Quels contours possède la norme mésolectale dont il est question ? Le français s'est plié à un certain nombre d'adaptations, comme notamment l'extension et l'augmentation du lexique ${ }^{12}$ dans les domaines de la vie quotidienne africaine (Schmitt 1984), distincte de celle de France. En revanche, lorsque je commençais mes recherches sur l'abidjanais, on m'a signalé à plusieurs reprises que sa syntaxe était largement conforme à celle du français de France et qu'il n'y aurait donc pas grand-chose à en dire, alors que personne ne l'avait encore réellement étudié. En résumé, on note qu'il y a des particularités plus égales que d'autres. Celles du niveau lexical et morpholexical ont été reconnues assez rapidement comme vecteurs d'une identité spécifique, alors que celles des niveaux syntaxique et morphosyntaxique restent reléguées au rang des barbarismes, ou, plus poliment, à celui de déviances de la norme, rapportées aux origines sociales (populaires) des locuteurs et réputées compromettre la communication inter-régionale. Audelà du standard, toujours rien à l'horizon ?

\title{
Côté locuteurs : identité sociale, nationale, francophone?
}

\author{
Insécurité linguistique
}

Au moment où le milieu scientifique en a déjà fait sa mascotte, le français populaire ne constitue pas encore ce vecteur de l'identité nationale qu'il deviendra par la suite : "La langue française nous sert dans nos manières d'agir, mais nos manières d'être restent soumises à nos langues africaines » explique un intellectuel ivoirien ${ }^{13}$. Rappelons que la subversion résulte du fait que la population se trouve exclue de la communication publique contre son gré ; il ne s'agit guère d'un refus délibéré de participer comme le suggérait Calvet (1974) par l'image de la langue qui devient «maquis » du peuple.

L'enquête de Pairault (1982) menée dès le début des années 1980 en milieu scolaire met en exergue le conflit individuel qu'implique l'adoption d'une langue seconde (pour la population étudiée, le contact avec le français a eu lieu lors de la scolarisation). Un enquêté déclare le plurilinguisme responsable de la déculturation : «Il m'a empêché d'avoir une maîtrise parfaite de ma propre langue. Je me rends compte, avec beaucoup d'aigreur et d'amertume, que je connais encore mal les arcanes de la pensée mythique, religieuse, l'organisation et la vie culturelle de mon propre milieu. » Et l'élève de conclure : "Pour tout dire, je me reconnais une personnalité construite avec du "matériau" étranger» (Pairault 1982: 90).

12. Se reporter notamment aux études lexicologiques de Lafage dans le cadre du projet IFA.

13. Cité par Duponchel (1979: 413), c'est lui qui souligne. 
À la même époque, l'enquête de Lafage (1980a) révèle que la majorité des jeunes Ivoiriens (cultivés, en l'occurrence) n'aspirent pas à parler français «comme les Français », alors qu'ils restent, somme toute, assez défavorables à l'accent ivoirien. Ce paradoxe peut s'expliquer par le fait que l'insécurité linguistique, double à l'origine (régionale et sociale), est tout juste en passe de se résoudre dans une insécurité purement sociale. On observe déjà une (relative) conscience du continuum qui s'installe : la quasitotalité des enquêtés estiment pouvoir deviner le niveau d'instruction et la catégorie socioprofessionnelle d'un interlocuteur selon sa façon de parler français. Dix ans plus tard, l'adoption semble consommée: l'enquête de Simard (1991) indique que le statut social d'un locuteur enregistré est souvent sous-évalué par le témoin interrogé — un banquier peut facilement être identifié comme boy. Cette tendance à dévaloriser un locuteur selon sa façon de s'exprimer, même sur des critères imaginaires, relève d'une conscience linguistique qui recoupe la conscience sociale avec un mépris des formes marquées considérées comme inférieures. Il s'agit d'un phénomène sans fondement linguistique, mais constitutif de toutes les communautés linguistiques.

\section{Identité nationale ou sociale?}

C'est donc à partir des années quatre-vingt que le français populaire (FPA/ FPI) prend les allures d'un modèle positif pour les Ivoiriens, pour devenir une vraie langue nationale (ou endogène) — chose alors inimaginable pour le français standard. Mais là aussi, de nombreuses ambiguïtés accompagnent la perception de ses contours.

Lafage (1980a) relate la perception et les jugements des étudiants de l'université d'Abidjan ${ }^{14}$ par rapport au FPI, lequel serait dans l'ensemble «plus utile que nuisible» (un Béninois), car «[il] vaut mieux ce français que rien du tout » (un Baule), pragmatisme qui défie la politique menée en faveur du français standard. En outre, la plupart des étudiants est favorable à l'emploi du FPI parce que celui-ci présente une image divertissante : la majorité des enquêtés lit les publications en FPI souvent ou très souvent, la plupart d'entre eux juge utile d'étudier ses caractéristiques en classe. Le FPI répond désormais aux besoins de communication intergroupe tout autant qu'aux besoins identitaires d'authenticité africaine des jeunes Ivoiriens instruits et occidentalisés.

Mais la vision du FPI n'est pas aussi unifiée qu'on le voudrait; un enquêté prétend que «ce français met le ministre au niveau du manœuvre,

14. Recueillis à travers une enquête auprès de 67 étudiants de deuxième année en Lettres, choisis dans les proportions ethniques similaires à celles du pays ; questions : les locuteurs « modèles » (enseignants et médias), la perception des différents registres, l'authenticité africaine (français standard/langues ethniques), l'attitude vis-à-vis du FPI. 
et tout Ivoirien le comprend sans même l'apprendre ». D'après ce témoignage, le FPI représente toute l'échelle sociale ivoirienne, et doit être suffisamment présent pour que la jeune génération grandisse déjà avec lui et le ressente comme une évidence. Lafage (1980a : 28) émet des doutes sur la variété désignée comme FPI par les étudiants :

«[...] entre eux, les étudiants emploient souvent le FPI mais [...] celui-ci semble relativement différent du parler véhiculaire des peu ou non-lettrés, plus caricatural. Les structures morpho-syntaxiques laissent plus ou moins poindre une norme sousjacente, seuls les traits les plus typiques (omission de l'article, présence d'un déterminant nominal postposé, séries verbales, conjugaisons plus ou moins neutralisées) y figurent. Par contre, la néologie lexicale y est abondante et souvent spirituelle. »

C'est en fait une variété acrolectale qui constitue le facteur d'intégration pour ces étudiants, tout en étant identifié au français populaire qui recouvre en fait une réalité linguistique distincte ; l'attitude face à la norme ne s'en trouve pas moins modifiée.

Selon Lafage \& Gadet (1995), on se dirigerait aujourd'hui vers la constitution d'un français «commun » local, avec le statut d'un français «très régionalisé », comparable à celui de Montréal. L'enquête sociolinguistique que j'ai menée en 1993 auprès des écoliers de deux quartiers populaires abidjanais (dont je rends compte plus en détail dans Ngalasso \& Ploog 1998), montre tout d'abord que l'emploi du «français » est généralisé, à travers toutes les situations de parole, avec tous les interlocuteurs; la pratique de la langue ethnique se restreint dans le meilleur des cas au cadre familial. Ensuite, on constate que si la conscience linguistique va en grandissant, quasiment tous les témoignages sont ambigus. Certains, qui, dans les échanges entre pairs, font preuve d'une habileté certaine, prétendent avoir appris le français à l'école ; d'autres, à l'évidence volontaires ou du moins curieux d'échanger avec moi, ne répondaient pas aux questions posées : inhibition ou incompréhension? Il s'agissait régulièrement d'enfants issus de l'immigration (deuxième génération) ou des milieux sociaux les plus bas : si la distance entre leur vernaculaire et le français requis à l'école empêche l'intercompréhension, il n'est que naturel qu'ils envisagent le français comme une entité étrangère que l'école leur a apportée. Bien qu'elle ne soit pas généralisable, la tendance à la rupture du continuum existe.

Les conclusions de Varoqueaux-Drevon (1995) sont plus optimistes. D'après son enquête ${ }^{15}$, les écoliers ivoiriens associent comme première habileté au français (sans autre distinction) le « parler» — tandis que la langue maternelle (ethnique) présente un taux plus important pour «écouter» et « entendre ». Les sentiments associés le plus fréquemment à la langue française sont la fierté, la sympathie, et le sérieux. Cette image très dynamique du français est cependant à nuancer; on établit sans difficulté le lien entre

15. Une fois de plus, il s'agit d'une enquête menée en milieu scolaire, avec des élèves du secondaire. 
ces appréciations et les arguments permettant de maintenir la pression du français sur les locuteurs ivoiriens, à savoir l'unité nationale avec son corollaire qui est l'identité ivoirienne et la croyance au progrès, sur laquelle elle est fondée. La comparaison avec les sentiments face aux langues maternelles s'impose : il ressort en effet des décomptes (Varoqueaux-Drevon 1995) que les appréciations individuelles sont plus révélatrices de l'attitude globale de l'enquêté que de sa relation spécifique avec la langue française. Il apparaît que ceux qui indiquent parler très bien le français parlent majoritairement très bien ou bien leur langue maternelle; de même, ceux qui indiquent parler mal le français ne parlent que moyennement leur langue maternelle. On constate ainsi un parallélisme assez régulier entre appréciation de la maîtrise de la langue maternelle et de celle du français, qui serait plutôt à décrire en termes de (in)sécurité linguistique individuelle générale. La corrélation avec la Norme du français serait à étudier : pourquoi ne pas imaginer que le crible scolaire déteigne sur la perception des pratiques langagières «tout court »?

Le point commun entre mon enquête de 1993 et celle de VaroqueauxDrevon réside dans le fait que la distinction entre le français standard et la variété locale n'est pas opérée par les enfants eux-mêmes, en tout cas pas spontanément. Dans la mesure où les problèmes de compréhension sont réels, et fréquents, il paraît inimaginable toutefois qu'ils ne réalisent pas « quelque chose ».

\section{Question d'étiquette}

\section{Discours d'observateur}

Comment décrire ce «quelque chose »? Deux dynamiques parallèles sont à distinguer: si les deux se ressemblent beaucoup aux yeux du locuteur non local, le FPA réfère à la grande époque des migrations et «stigmatise » aujourd'hui les locuteurs âgés (d'après Lafage \& Gadet (1995), et mes enquêtes tendent à le confirmer); ensuite, une élaboration formelle a eu lieu au cours des trente années passées ${ }^{16}$. Ces restructurations ne peuvent plus être interprétées comme stratégies d'apprentissage d'une langue seconde puisque l'ex-FPA est le parler ordinaire - de plus en plus souvent la première langue acquise — des enfants abidjanais des années quatrevingt-dix. Le FPA a initié un processus de changement linguistique, amorcé par la génération des migrants et confirmé par leurs descendants, les premiers à adopter le FPA comme variété «à tout faire». Les pratiques de la jeune génération se différencient de celles de la génération précédente du point de vue à la fois formel et socio-interactionnel.

16. J'en rends compte dans Ploog (1999a, 1999b). 
J'ai pris le parti de nommer ce nouvel objet abidjanais, terme qui n'existe ni dans la réalité locale, ni dans la littérature scientifique. Depuis toujours, il est identifié comme étant du français — à tort ou à raison —, étiquette à laquelle on a collé des épithètes variées. Passé une première phase de créations éphémères (Kokora 1983; Duponchel 1979; Lafage 1978b; Partmann 1981; Manessy 1992) — français de Treichville, du marché, des Burkinabè, des petits métiers —, deux caractéristiques sont récurrentes dans les dénominations. Lorsqu'on le décrit comme français régional, on lui reconnaît l'attachement à un territoire, tout en notant son caractère oral et marginal ; la dénomination du FPA a évolué dans le sens où il est devenu FPI et actuellement on assiste à l'émergence de l'appellation français des rues, sous-jacente depuis longtemps; en dehors du fait que les rues ne parlent guère, il me semble réducteur de limiter l'emploi de la sorte. Lorsqu'on le nomme français populaire, on lui reconnaît une communauté de locuteurs, tout en le discréditant comme émanant des basses couches sociales.

Le présupposé commun à tous les termes employés consiste à opposer la langue et les dialectes : dans l'usage commun, le dialecte est une variante simple de la langue, appréciation qui ne se prive pas d'y intégrer la simplicité des locuteurs. Au sens saussurien, le dialecte est un sous-ensemble de la langue; avec toutes les restructurations qu'il a subies, l'abidjanais estil encore un sous-ensemble du français ? Si, pour d'autres terrains, on a plus récemment proposé des dénominations plus appropriées comme le français ordinaire (Gadet 1989) ou le français parlé (Blanche-Benveniste \& Jeanjean 1987), notre «quelque chose » attend toujours le grand soir.

\section{Discours de locuteurs}

En tant que locuteurs, comment évaluent-ils ce qu'ils parlent ? J'avais veillé, dans mes questions, à ne pas introduire une segmentation artificielle qui risquait de créer sur leurs témoignages des distorsions dont l'impact me paraissait trop difficile à maîtriser. De mes enquêtes dans le milieu scolaire ${ }^{17}$ ressortent deux grandes attitudes face à l'entité linguistique mouvante qui les entoure :

- il y a le français d'un côté et ma/notre pratique quotidienne (qui ne porte pas de nom) de l'autre; il faut s'efforcer de parler français avec les personnes qu'on respecte;

- il y a le bon et le mauvais français : le mauvais français c'est «quand on parle entre nous et on dit "epi-epi-epi" » (élève de CE1, enquête 1993) ; il faut s'efforcer de parler du bon français avec les personnes qu' on respecte.

17. Celle de 1993, spécifiquement orientée vers les écoliers, et celle de 1997, à visée plus large, qui s'adressait aux populations scolaires et aux enfants de la rue (= citadins natifs de moins de 15 ans). 
Les comportements qui en résultent respectivement se ressemblent en tout point ; celui qui ne se sent pas apte à produire du (bon) français se tait.

Mon terrain auprès des enfants de la rue confirmerait exclusivement la seconde attitude : ce qu'ils parlent, c'est du français. Le paramètre distinctif le plus révélateur semble être la pression normative subie par le locuteur au moment où il doit s'exprimer, de loin moins importante chez les enfants de la rue, qui ne se laissent pas aussi facilement empêcher de parler. Par ailleurs, les enfants de la rue ne sont pas dégagés non plus de toute pression normative (Ploog 2000) : nombreux sont les cas d'hypercorrections, de discours « du dimanche » en situation formelle telle l'interview, face-à-face avec l'enquêtrice : ce qu'ils prennent pour l'acrolecte est un mésolecte qu'ils ne maîtrisent pas. Mais s'ils sont beaucoup moins inhibés, ils ne nomment pas non plus leur parler ordinaire. Une seule fois, un enfant qualifie une expression de «français de bakroman » : bakroman est une création hybride diula-anglais par laquelle les enfants de la rue se désignent eux-mêmes ; il faut le comprendre ici comme référence à un dérivé de l'argot nouchi, qui ne se superpose pas à la pratique quotidienne.

Bien que le mauvais français semble un terme spécifique aux écoliers, pour les autres, il n'y a point de salut hors de l'école non plus. Voici l'explication d'un enfant de la rue (11 ans, déscolarisé, rescolarisé depuis peu) qui résume le paradoxe abidjanais dans toute sa splendeur (portion d'enquête I.06 : $080-112)^{18}:$

Enquêtrice: «Où est-ce que tu as appris le français ?

Locuteur 1: - C'est à Bingerville quand j'étais petit parce que j'ai fait le CP1, CP2, CE1, et après le CE2.

$\mathrm{E}$ : $\quad-$ Donc tu l'as appris à l'école; à la maison vous parlez quoi avec tes parents?

L1: $\quad$ - Parfois on parle - parce que je suis ebrie - parfois on parle l'ebrie, parfois on parle le français; mais moi je parle pas l'ebrie, quand on parle en ebrie, je réponds en français.

$\mathrm{E}: \quad-\mathrm{Tu}$ comprends l'ebrie?

L1 : - Oui mais pas trop pas trop ; maintenant, j'ai commencé à oublier, j'ai oublié beaucoup de mots [...].

$\mathrm{E}: \quad-$ Ça vous dérange pas que vous parlez pas votre langue ethnique?

L1: $\quad-$ Non, moi ça me dérange pas parce que chez moi, quand je parle l'ebrie avec mes [parents] ebrie, j'ai honte (Pourquoi ?) parce que à l'école, on m'a pas appris à parler l'ebrie, on m'a surtout appris à parler le français. Donc quand je parle l'ebrie, surtout avec mes parents, j'ai honte. Donc j'ai laissé tomber, je parle le français.

18. Les propos ont été traduits. 
E : - Et ce serait pas bien de parler l'ebrie à l'école ?

L1 : $\quad$ - Non (Pourquoi ?) parce que tous mes vieux ${ }^{19}$ parlent français, et [si] moi je parle ebrie avec mon ami, ça fera pas beau. [...] Si tous les enfants parlaient l'ebrie à l'école, ça ferait pas beau parce que, premièrement, quelqu'un qui ne comprend pas l'ebrie, qui ne parle que le français, sera obligé de répondre en ebrie, alors qu'il ne comprend pas l'ebrie. Donc c'est pour ça qu'on a fait l'école : pour que tout le monde parle français.

E : $\quad$ - Donc l'école, c'est pour que tout le monde parle français ?

L1 : - Oui. Ceux qui ne comprennent pas bien le français.

E : — Ils vont à l'école ? (Oui) Mais ceux qui parlent français, ils n'ont pas besoin d'aller à l'école ?

L2 : $\quad-\mathrm{Si}$, on apprend...

L1: $\quad-$ Si, parce que il y a des choses qu'on ne comprend pas. Ils savent parler mais ils ne savent pas ce que ça veut dire. Par exemple, à l'école : "bonjour" (mh) ils ne savent pas ce que ça veut dire ; chez eux, ils se saluent "bonjour, bonjour". Alors "bonjour", ça veut dire "je te salue" en français, mais chez eux ça veut dire "bonjour", c'est "bonjour". Donc ils savent pas ce que ça veut dire. Donc c'est pour ça qu'on a fait l'école : pour pouvoir comprendre ce que tu dis, et pour apprendre le français. »

Bref, avant d'arriver à l'école on parle (le français ?), et à l'école on apprend (le français !). Dans le même ordre d'idées, un autre enfant, celuilà non scolarisé, justifie son parler par son efficacité - il est vrai que l'apprentissage, il ne pourrait pas en justifier — vis-à-vis d'un éducateur qui les reprend (portion d'enquête V.04/170-175) :

«---_20 il coude dans mon ventre !

- Il fait quoi ?

- Il coude m--

- Il [ku]?

- Il coude.

— Il t'a coudé dans le ventre, ça se comprend (quand même) ! ${ }^{21}$.

Mise à part la coïncidence de mon enquête, rien ne rapproche ces deux locuteurs. Et pourtant, le ténor du discours, la double base de la pratique extrascolaire (les normes) et des justifications (la Norme), indique une communauté linguistique bien réelle.

19. Les adultes de la famille, ou les aînés.

20. Portion de discours inaudible.

21. Le terme kud signifie «donne des coups de coude»; le caractère plus ou moins spontané de cette dérivation reste à établir. 
La différenciation des variétés est loin d'aller de soi. La seule constante dans les délimitations d'une variété locale, abidjanaise ou ivoirienne, ou de toute autre sous-catégorisation proposée, est que la description entraîne une profusion de guillemets autours des dénominations, ceci en partie à cause des implications sociopolitiques qui pèsent sur tous, citoyens et locuteurs autant que linguistes. Changement et insécurité résultent tous deux de l'appropriation. Ce n'est pas le changement lui-même qui génère l'insécurité, ni l'emplacement de l'idiolecte sur l'axe du continuum ; d'après mes observations, elle résulte plutôt du positionnement du locuteur face au continuиm, qu'il conduit le cas échéant à entretenir. La dynamique n'est pas linguistique, mais sociale, tout comme le rapport de force qui l'anime.

Autant que les locuteurs, les linguistes procèdent à un nivellement par le haut, ce qui laisse de côté ce que l'abidjanais possède de plus original: son hétérogénéité, qui rend le travail même du linguiste périlleux, ou, du moins, qui crée de fait une inhibition. Si la norme endogène existe, elle possède une articulation multiple; il faut ainsi accepter une description par l'hétérogène. Mais l'objectif des recherches autour de la norme endogène du français en Afrique est la reconnaissance de celui-ci au même titre que le français du Québec, de Belgique, et de Suisse; cette reconnaissance comprend la complémentarité - et non la concurrence — des diverses variétés du français coexistant en Afrique. Néanmoins, la présentation d'une variété proche du français standard comme mésolectale participe à la consolidation de l'emprise française sur l'Afrique, qui revient à une appropriation de la problématique. Ceci n'est pas sans rappeler le conflit précédent, entre français et langues ethniques.

\section{Post-scriptum}

Le problème pour les linguistes est plus que terminologique, car, comme le souligne Cécile Canut (ici-même), nommer c'est instituer socialement. J'avais pris le parti d'attribuer à mon objet l'étiquette abidjanais pour prendre mes distances par rapport aux dénominations antérieures qui me paraissaient problématiques. En le nommant, je m'expose aux mêmes critiques que celles que je viens de formuler, et je ne voudrais pas donner l'impression de me placer au-dessus de la polémique.

Si je l'ai nommé, c'est parce qu'on ne peut éviter de nommer son objet d'analyse. Je maintiens aujourd'hui cette étiquette mais pour une raison distincte, plus fondamentale me semble-t-il. Lorsque la variabilité des usages empêche par moments l'intercompréhension avec la francophonie extérieure, et lorsqu'il n'y a plus guère de certitudes qui justifieraient une dénomination quelconque en référence aux locuteurs ou à leur appartenance sociale, celle qui prend appui sur le lieu a le mérite de décrire de façon 
objective le contexte d'observation, dont la spécificité même justifie de le distinguer d'autres contextes : une appellation d'origine n'est pas en soi un label de qualité. Dans cette perspective (extérieure), l'étiquette abidjanais me paraît la moins mauvaise.

Pour ce qui est des locuteurs, ce n'est pas parce qu'ils ne le nomment pas qu'ils ne catégorisent pas. Leur catégorisation toutefois n'est pas monolithique. Si leur identité même est composite, il n'est pas surprenant que leur parler le soit - et qu'il ne porte pas de nom. Après tout, il n'y a pas de raison de nommer l'évidence ; il n'y a que l'observateur qui relève cette particularité qui a besoin de la nommer. Au-delà, l'abidjanais n'existe pas.

Équipe de recherche en syntaxe et sémantique, Université Bordeaux 3.

\section{BIBLIOGRAPHIE}

Blanche-Benveniste, C. \& Jeanjean, C.

1987 Le français parlé. Transcription et édition, Paris, CNRS/INALF.

CAlvet, L.-J.

1974 Linguistique et colonialisme. Petit traité de glottophagie, Paris, Payot («Bibliothèque scientifique »).

Chaudenson, R.

1989 «Proposition pour une grille d'analyse des situations linguistiques de l'espace francophone », in R. CHAUdENSON \& D. DE RoBILLARD, eds, Langue, économie et développement, 2 vols, Aix-en-Provence, Institut d'études créoles et francophones, Université de Provence : 201-257.

1993 «Francophonie, "français zéro" et français régional», in M. BENIAMINo \& D. DE ROBILlaRD (dir.), Le français dans l'espace francophone : description linguistique et sociolinguistique de la francophonie, 2 vols, Paris, Honoré Champion : 385-405.

Crespo-Meunier, M.

1991 Des données empiriques à l'objet d'étude: la constitution d'un corpus de démodialectologie (le français populaire d'Abidjan), Thèse nouveau régime, Lyon, Université de Lyon 3.

1998 «La labilité morphologique et la notion de diamorphe», in A. QuefFéLEC, ed., Alternances codiques et français parlé en Afrique. Actes du colloque d'Aix-en-Provence, septembre 1995, Aix-en-Provence, Publications de l'Université de Provence : 307-309.

DeRIVE, M. J.

1986 «Francophonie et pratiques linguistiques en Côte-d'Ivoire », Politique africaine, $23: 42-56$. 
Dubresson, A. \& Yapi-Diahou, A.

1988 «L'État, "le bas", les cours : exclusion sociale et petite production immobilière à Abidjan (Côte-d'Ivoire) », Tiers Monde, 116:1083-1100.

DuMONT, P.

1991 Le français, langue africaine, Paris, L'Harmattan.

1993 «L'enseignement du français en Afrique : le point sur une méthodologie en crise », in M. Beniamino \& D. DE Robillard (dir.), op. cit. : 471-482.

DUPONCHEL, L.

1971 «Multilinguisme et français scolaire chez l'écolier ivoirien », Bulletin de Liaison CURD, $1: 13-18$.

1974 «Le français d'Afrique noire : mythe ou réalité ? Problèmes de délimitation et de description», Annales de l'Université d'Abidjan, série H, VII-1 : 133-158.

1979 «Le français en Côte-d'Ivoire, au Dahomey et au Togo», in A. VALDMAN (dir.), Le français hors de France, Paris, Honoré Champion : 385-418.

GADET, F.

1989 Le français ordinaire, Paris, A. Colin («Linguistique»).

HATTIGeR, J.-L.

1983 Le français populaire d'Abidjan : un cas de pidginisation, Abidjan, Université d'Abidjan, Publication de l'Institut de linguistique appliquée.

KOKORA, P.

1983 «Pourquoi parle-t-on tant de la promotion des langues nationales dans le système éducatif ? », Cahiers ivoiriens de Recherche linguistique, 13 : 95146.

LAFAGE, S.

1978a «Description sommaire de la situation sociolinguistique en Côte-d'Ivoire », Cahiers ivoiriens de Recherche linguistique, 3: 7-78.

1978 b « Rôle et place du français dans le continuum langues africaines-français en Côte-d'Ivoire », Cahiers ivoiriens de Recherche linguistique, 4 : 54-66.

1980a «Petite enquête sur la perception du français populaire ivoirien en milieu estudiantin », Bulletin du Centre d'études sur le plurilinguisme, 6/80 : 1-37.

1980b « Enquête sociolinguistique en Côte-d'Ivoire », Bulletin de l'Observatoire du français contemporain en Afrique noire, 1: 13-16.

1984 «Note sur un processus d'appropriation socio-sémantique du français en Côte-d'Ivoire », Cahiers de l'Institut de linguistique de Louvain, 9/3-4: 103-112.

1991 «L'argot des jeunes Ivoiriens, marque d'appropriation du français?», Langue française, 90 : 95-106.

LAFAGE, S. \& GADET, F.

1995 «Sur le terrain: interview de Suzanne Lafage (Paris III)», LINX, 33 : 101-109. 
MANESSY, G.

1990 «Modes de structuration des parlers urbains », in R. CHAUDENSON, ed., Des langues et des villes, Paris, ACCT/Didier Érudition : 7-23.

1992 «Normes endogènes et normes pédagogiques en Afrique noire», in D. BAGGioni et al., eds, Multilinguisme et développement dans l'espace francophone, Paris, ACCT/Didier Érudition : 43-75.

1994 « Normes endogènes et français de référence », in G. MANESSY, ed., Le français en Afrique noire: mythes, stratégies, pratiques, Paris, L'Harmattan: 215-227.

MARGUERAT, Y.

1979 Des ethnies et des villes : analyse des migrations vers les villes de Côted'Ivoire, Abidjan, Orstom.

Ngalasso, M. M. \& Ploog, K.

1998 «Le français des écoliers abidjanais : la revanche de la rue sur l'école?», in A. BATIANA \& G. PRIGNitz, eds, Francophonies africaines, Rouen, CNRS/ Université de Rouen : 49-65.

PAirault, C.

1982 «À propos de la langue maternelle en Afrique noire», Langue française, $54: 85-92$.

PartmanN, G. H.

1981 «L'identification nationale et l'ivoirisation du français », Bulletin de l'Observatoire du français contemporain en Afrique noire, 2: 13-22.

PloOG, K.

1999a Le premier actant en abidjanais. Contribution à la syntaxe du non-standard, Thèse de doctorat, Bordeaux, Université de Bordeaux 3.

$1999 b$ « Turbulences dans la zone préverbale : sujet- $\varnothing$ et conjugaison objective en français d'Abidjan », Le Français en Afrique. ROFCAN, 13 : 105-116.

2000 «La Norme dans l'observation des normes abidjanaises : étude d'un continuum linguistique », Lengas, $48: 103-128$.

Schmitт, C.

1984 «L'emprunt du français aux langues africaines », Cahiers de l'Institut de linguistique de Louvain, IX (3-4) : 203-216.

SENGHOR, L. S.

1988 Ce que je crois: négritude, francité et civilisation de l'universel, Paris, Grasset.

SIMARD, Y.

1991 « Principes et méthodes pour l'enquête en Côte-d'Ivoire », Bulletin du Centre d'études sur le plurilinguisme, Institut d'études et de recherches interethniques et interculturelles, 12/91: 5-15.

1994 «Les Français de Côte-d'Ivoire », Langue française, 104 : 20-36. 
TABI-MANGA, J.

1992 «Le français d'Afrique (bilan, évaluation et stratégie prospective) », Études linguistique appliquée, 88 : 111-116.

VARoqueauX-Drevon, I.

1995 « Sentiments et comportements linguistiques. La représentation de la langue française en tant que langue de scolarisation en Côte-d'Ivoire », Cahiers des Sciences humaines, XXXI (1) : 83-101.

\section{RÉSUMÉ}

La constellation plurilingue ivoirienne se distingue de celle rencontrée dans d'autres anciennes colonies françaises. Dans un contexte de brassage ethnique, d'illettrisme et d'urbanisation intenses, le français n'est pas resté le "supervéhiculaire »qu'il est ailleurs : après avoir été plébiscité comme véhiculaire urbain, tout en subissant des adaptations structurales majeures, il est investi désormais de fonctions vernaculaires. Les locuteurs semblent avoir une perception à géométrie variable de ce qu'ils pratiquent; les descriptions linguistiques de cette entité, elles, sont très variées. Tous conviennent cependant du fait qu'il s'agit de français, appréciation déconcertante pour le francophone non local, pour qui grand nombre d'échanges locaux restent opaques. II apparaît que la volonté de délimitation d'une variété ou de toute autre sous-catégorisation des formes est vouée à l'échec : en effet, elle réduit l'appropriation à un changement de la langue en négligeant les effets produits sur une communauté vivant dans une langue mouvante. Par ailleurs, cette hétérogénéité intrinsèque de l'objet semble mettre en cause le travail même du linguiste.

\section{ABSTRACT}

The Polemic of Abidjanee "French": a Non-Standard Variety between Endogenous Norm and the Fantasy of Uniqueness. - In the Ivory Coast, more than in any other of France's ex-colonies, the official language-which is French-has undergone some reorganization during the last fourty years, which has acquired some notoriety in the scientific world during the seventies. In our day, Abidjanee is a linguistic continuum which engenders a wide range of structures. In fact, a (non-local) native speaker of French often cannot understand Abidjanees, while they maintain that they speak French. The most important social factor of this process is the urban environment, with its heterogeneity, density, competition, and the lack of norm in the linguistic model. Abidjanee popular French (FPA in French) has been described as a pidgin, pre-creole language, or regional variety. Nevertheless, it is definitely impossible to distinguish a homogeneous Abidjanee variety from (standard) French without any regard to the speakers intuition, that shows a variable perception of the diversity encountered in their speech-and of its social meaning.

Mots-clés/keywords: Côte-d'Ivoire, appropriation, continuum linguistique, dynamique urbaine, français, identité, Normes, représentation/Ivory Coast, appropriation, linguistic continuum, urban dynamics, french language, identity, Norms, representation. 